\title{
Synthesis And Characterization of Thermo-Responsive Particles of Poly(Hydroxybutirate-Co-Hydroxyvalerate)-B-Poly(N-Isopropylacrylamide)
}

\author{
Adriano Marim de Oliveira \\ Department of Chemical Engineering, School of Engineering of Lorena-University of São Paulo, Lorena - SP - Brazil and \\ Institute for Technological Research of State of São Paulo - SP - Brasil \\ Pedro Carlos de Oliveira and Amilton Martins dos Santos* \\ Department of Chemical Engineering, School of Engineering of Lorena-University of São Paulo, Lorena - SP - Brazil \\ Maria Helena Ambrosio Zanin and Maria Inês Ré \\ Institute for Technological Research of State of São Paulo - SP - Brasil
}

(Received on 15 July, 2008)

\begin{abstract}
A new kind of thermo-responsive particles were prepared by the self-assembly technique, comprising poly(hydroxyvalerate-co-hydroxybutirate)-b-poly(N-isopropylacrylamide)/ (PHBHV-b-PNIPAAm) block copolymers. The hydrophilic part PNIPAAm was synthesized by Reversible Addition- Fragmentation chain Transfer (RAFT) polymerization. Particles with core-shell morphology were obtained with hydrophilic outer shells and hydrophobic inner cores. Dexametasone acetate (DexAc) was used as a model drug with an encapsulation efficiency of $77 \%$. The release of DexAc in aqueous solution was strongly dependent on temperature, suggesting that PHBHV-b-PNIPAAm particles can be used as a thermo-responsive carrier material with external control in a drug release system.
\end{abstract}

Keywords: thermo-responsive particles, dexametasone acetate, drug release, block copolymer, RAFT

\section{INTRODUCTION}

Stimuli-responsive polymers are defined as polymers that undergo relatively large and abrupt physical or chemical changes in response to small external changes in the environmental conditions. There are many different stimuli to modulate the response of polymer systems. These stimuli can be classified as either physico-chemical or biological [1]. Ionic strength, $\mathrm{pH}$ and chemical agents can be considered as chemical stimuli, whereas temperature, electric or magnetic fields and mechanical forces are pointed out as physical stimuli [2]. Temperature and $\mathrm{pH}$ are the most widely used stimuli in environmental responsive polymer systems. The change of temperature is not only relatively easy to control, but also easily applicable for in vitro and in vivo drug delivery experiments [3].

Thermo-sensitive polymers, such as PNIPAAm and their copolymers, have been well-exploited in drug delivery systems [4]. PNIPAAm is a water-soluble polymer exhibiting an extended chain conformation below the Lower Critical Solution Temperature (LCST) of $32^{\circ}$, and coalescing above this temperature forming hydrophobic and insoluble aggregates $[5,6]$.

Polyhydroxybutirate (PHB), a biodegradable polymer, and its copolymers can be used as raw materials for many applications due to their physical-chemical and biocompatible characteristics [7].

Previous works have shown the utilization of PHB or its copolymers in drug delivery systems [7, 8].

Recently, self-assembly technique have provided an efficient and rapid pathway for the synthesis of objects in the nanometers to micrometers range, which are difficult or even impossible, to be obtained by conventional chemical reac-

\footnotetext{
*Electronic address: amsantos@dequi.eel.usp.br
}

tions. A recent approach looks into the possibility of reducing tedious and time consuming synthetic steps by engineering oligomers or polymers of relatively small size that can self-organize by only physical forces, simulating the folding of peptides segments in proteins $[9,10]$. Among the various aggregation processes, the most extensively studied concerns the self-assembly of block or graft copolymers. Amphiphilic block copolymers have been used extensively due to the properties of the self-assembling micelles formed in the presence of a suitable solvent. In an aqueous environment, the hydrophobic parts of the block copolymers form the core of the micelle, while the hydrophilic ones form the corona or outer shell. The hydrophobic micelle core acts as a microenvironment for the incorporation of lipophilic drugs, while the corona shell works as a stabilizing interface between the hydrophobic core and the external medium [11-14].

Block copolymers consisting of a PNIPAAm segment and a hydrophobic segment can form core-shell micelle structure below the LCST. This polymeric micelle structure comprises a hydrophilic outer shell of hydrated PNIPAAm segments and a hydrophobic inner core. The inner core can be loaded with hydrophobic drugs, while PNIPAAm outer shell plays the role of aqueous solubilization and temperature responsiveness. The hydrophilic outer shell, which prevents the inner core from interacting with biocomponents and other micelles, can suddenly become hydrophobic in specific conditions depending on the temperature [13,15-20].

In this work a block copolymer was synthesized by coupling reaction between PHBHV and PNIPAAm using N,N'-dicyclohexylcarbodiimide (DCC) and 4dimethylaminopyridine (DMAP) as coupling agents. The block copolymer was characterized by ${ }^{1} H$ NMR and Gel Permeation Chromatography (GPC). The thermo-responsive nanoparticles were prepared by dialysis method and analyzed by light scattering (LS) and transmission electron microscopy (TEM). The thermo-responsive behavior of nanoparticles was studied by turbidimetry and LS. Additionally, nanoparticles 
were loaded with Dexametasone acetate (DexAc) and the drug release behavior was determined in vitro in order to evaluate its application in drug delivery systems.

\section{EXPERIMENTAL}

\subsection{Materials}

$\mathrm{N}$-isopropylacrilamide (NIPAAm, 97\% from Aldrich) was used after purification through recrystallization in a mixture of 60:40 toluene/hexane. 2,2'-Azobisisobutyronitrile (AIBN) was purified by recrystallization in absolute ethanol. The RAFT agent (2-((2-Phenyl-1-thioxoethyl)thio)propanoic acid (PTET PA) was synthesized according to the methodology described in the literature [21]. N,N-dicyclohexyl carbodiimide - DCC (Aldrich, 99\%) and 4-dimethyl aminopyridine - DMAP (Aldrich, 99\%) were used as received. The solvents chloroform (Anidrol, 99\%), tetrahydrofuran (THF) (Vetec, 99\%), diethyl ether (Anidrol, 99\%), methanol (Anidrol, 99\%), dimethylformide (DMF) (Vetec, 99\%) and 1,4-dioxane (Anidrol, 99\%) were used as received. Dexametasone acetate (DexAc), was kindly furnished by Henrifarma and utilized without further purification. The PHBHV used in this work was supplied by PHB Industrial S.A. After the reduction of the molecular weight by means of a methodology described in the literature [22], the resulted PHBHV was characterized by $1 \mathrm{H}$ NMR using chloroform as solvent and Gel Permeation Chromatography (GPC) in a Waters equipment, with a 1515 isocratic HPLC pump; a 717 plus autosampler and a 2414 refractive index detector. A set of three phenogel columns (Labtron Phenomenex), with porosity of 120 A, 250 Aand 500 Awas used in the analyses. HPLC grade chloroform was used as eluent. The analyses were carried out using a flow rate of $1 \mathrm{~mL} / \mathrm{min}$, at $30^{\circ} \mathrm{C}$. The results obtained for PHBHV were: $M n=7973 \mathrm{~g} / \mathrm{mol}$ and $P I=2.53$.

\subsection{Synthesis and characterization of PNIPAAm}

The NIPAAm polymerization was performed in a glass reactor capped with a septum. The reaction vessel was loaded with 1,4-dioxane, NIPAAm ( $2 \mathrm{M})$, the RAFT agent (2-((2-Phenyl-1-thioxoethyl)thio) propanoic acid (PTETPA) $(5,0 \mathrm{mmol})$, and AIBN in a 4:1 [RAFT]/[AIBN] molar ratio. The mixture was purged with nitrogen for $20 \mathrm{~min}$ at $4^{\circ} \mathrm{C}$ The temperature was then raised to $70^{\circ} \mathrm{C}$ using a thermostated water bath. Polymerization was carried out under a nitrogen atmosphere for 5 hours. PNIPAAm was precipitated out with diethyl ether and recovered by filtration. PNIPAAm was characterized by ${ }^{1} H$ NMR (Varian, 300MHz) using chloroform as solvent. For this polymer the GPC analysis was performed using a $0.25 \%(\mathrm{w} / \mathrm{v})$ solution of tetrabutylammonium bromide in tetrahydrofuran (HPLC grade) as eluent, at the same conditions of flow rate and temperature, as was used in the analysis of PHBHV. The results for PNIPAAm were: $M n=35282$ $\mathrm{g} / \mathrm{mol} M W D=1.36$.
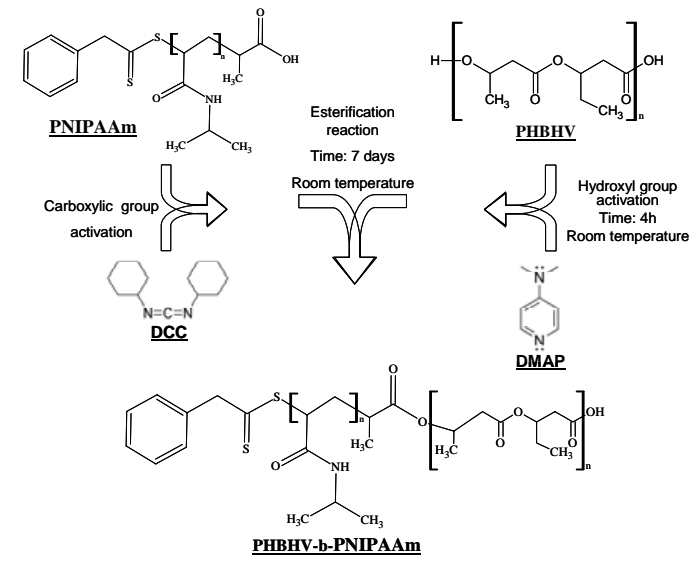

Scheme 1: Synthesis of PHBHV-b-PNIPAAm block copolymer

\subsection{Synthesis and characterization of PHBHV-b-PNIPAAm}

The block copolymer PHBHV-b-PNIPAAm, was obtained reacting hydroxyl-caped PHBHV with carboxyl-caped PNIPAAm (Scheme 1), as follows: PHBHV $\left(2.3 \times 10^{-4} \mathrm{~mol}\right)$ and DMAP $\left(1.5 \times 10^{-3} \mathrm{~mol}\right)$ were dissolved in $50 \mathrm{~mL}$ of chloroform under nitrogen and agitation, at room temperature. After 4 hours, PNIPAAm $\left(6.0 \times 10^{-6} \mathrm{~mol}\right)$ and DCC $\left(9.2 \times 10^{-4}\right.$ mol) were dissolved in $50 \mathrm{~mL}$ of chloroform and added to the reaction medium. This reaction was carried out during 7 days at room temperature. After this time the block copolymer was precipitated in an excess of cold methanol. The block copolymer was characterized by ${ }^{1} H$ NMR and by GPC using a Viscotek, model 302 Triple Detector. A 0.02 M0.02 M LiBr solution in N,N'-Dimhetylformamide (DMF) was used as eluent. The analysis was performed with a flow rate of 1 $\mathrm{mL} / \mathrm{min}$, at $60^{\circ} \mathrm{C}$.

\subsection{Preparation and characterization of nanoparticles}

PHBHV-b-PNIPAAm block copolymer was dissolved in DMF and dialyzed in water at room temperature using dialysis bag with a cut-off of 6,000-8,000 Da, for 24 hours.

\subsubsection{Particles size}

The particles size and particles size distribution were determined by 1000 , in aqueous medium.

\subsubsection{Particles morphology}

A drop of the particle suspension was placed on a copper grid and dried before analysis by TEM, in a Philips CM120 transmission electron microscope, at an acceleration voltage of $80 \mathrm{kV}$ (Centre Technologique des Microstructures (CT), Claude Bernard University, Lyon, France). 


\subsubsection{Optical characterization of particles aqueous suspension}

Optical characterization of PHBHV-b-PNIPAAm particles in aqueous suspension was carried out using a Turbscan Lab (Formulation, France) equipment. The particle suspensions were transferred to a glass cylindrical cell and analyzed by a light beam, emitted in near infrared light source $(880 \mathrm{~nm})$, which scanned the sample cell from bottom to top. Two synchronous optical sensors, transmission (T) and backscattering (BS), received the light that goes across the sample and that scattered backward, respectively, by the sample. The samples in the cell were scanned at two different conditions, heating from 25 to $60^{\circ} \mathrm{C}$ and cooling from 60 to $25^{\circ} \mathrm{C}$, for 24 hours. The change in transmittance as a function of time was taken as a measure in order to evaluate the thermo-responsive properties of nanoparticles.

\subsection{Drug loading}

PHBHV-b-PNIPAAm block copolymer (11.8 mg) and DexAc (10.3 mg) were dissolved in $1 \mathrm{~mL}$ of DMF and added, drop by drop, in $40 \mathrm{~mL}$ of water and dialyzed against water at room temperature. After dialysis, the DexAc-loaded particles were separated and frozen by a freeze dryer system (Liobras, L101). The encapsulation efficiency was calculated using the following equation:

$$
\% E=\frac{A c_{N}}{A c_{I}} \times 100
$$

Where $A c_{N}$ is the mass of drug in nanoparticles after liophilization obtained by UV/vis spectrometer, at $260 \mathrm{~nm}$ and $A c_{I}$ is the initial mass of drug.

\subsection{Drug delivery assay}

The release experiments of DexAc loaded particles were conducted in a buffer solution ( $\mathrm{pH} 7.4)$ at temperatures of 4 and $4^{\circ} \mathrm{C}$. PHBHV-b-PNIPAAm particles loaded with DexAc (1 mg) were placed in a dialysis bag (cut-off 6,000-8,000 Da) and immersed in $200 \mathrm{~mL}$ of the buffer solution. Samples were withdrawn for analyses of DexAc in the external buffer solution in a UV/vis spectrometer, at $242 \mathrm{~nm}$, in a time-course procedure.

\section{RESULTS AND DISCUSSION}

The synthesis of block copolymer with biodegradable segments occurred by means of the reaction between the hydroxyl end-groups of PHBHV and the carboxyl end-groups of PNIPAAm. The carboxyl-caped PNIPAAm was obtained using a functionalized chain transfer agent (PTETPA). This reaction was successfully conducted due to the use of DCC and DMAP as coupling agents. Figure 1 shows the ${ }^{1} H$ NMR spectra of PHBHV-b-PNIPAAm. The analysis of this spectrum allows identifying all signals of both segments, PHBHV and PNIPAAm, confirming the formation of the block copolymer.

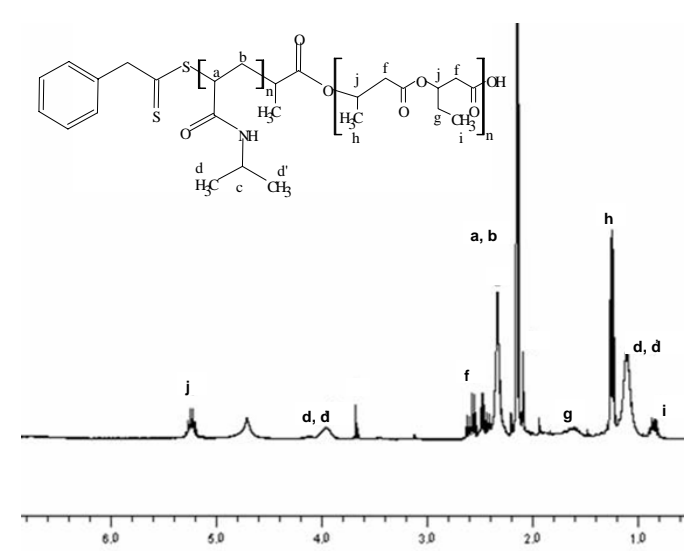

FIG. 1: ${ }^{1} \mathrm{H}$ NMR spectra of PHBHV-b-PNIPAAm

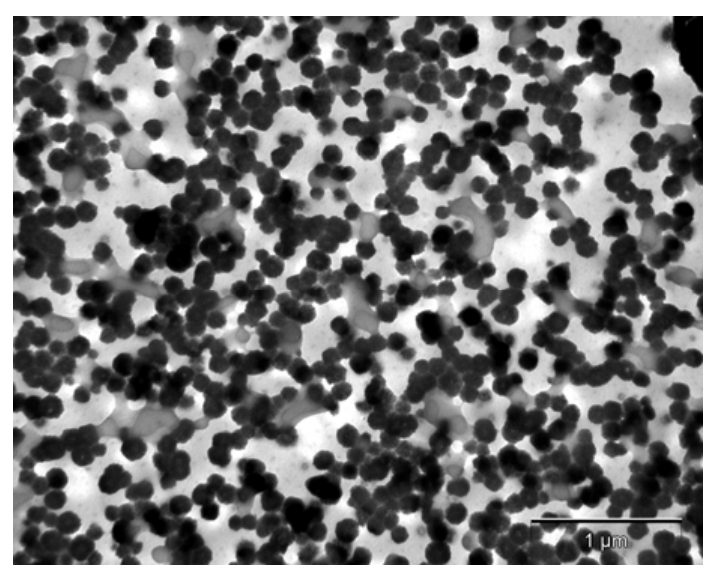

FIG. 2: TEM image of PHBHV-b-PNIPAAm nanoparticles.

These images were used to qualitatively verify the presence of particles of different sizes. This qualitative analysis corroborates the results obtained by LS, where an average particle diameter of $511 \mathrm{~nm}$ and a high polydispersity index (PI = 0.3 ) were observed. Additional and very important information from the TEM analyses is the particles morphology. An accurate analysis of Figure 3 shows the presence of particles with a core-shell morphology, more precisely, a PNIPAAm low contrast (green in the picture) outer shell and a dark inner core constituted of PHBHV segments. This finding suggests that PNIPAAm has stabilized the nanoparticles in the aqueous medium and turned these particles thermo-responsive.

To evaluate the instability of the dispersions, we used the transmission profiles produced by Turbiscan Lab equipment. The profiles are related to particle migration (creaming and sedimentation) and to the increase in particle size (coalescence and flocculation). Figure 4 shows the variation in the transmittance $(\Delta \mathrm{T})$ of an aqueous particle dispersion, as a function of a heating process (from $25^{\circ} \mathrm{C}$ to $60^{\circ} \mathrm{C}$ ) for 1 day, whereas Figure 5 shows the cooling process of the same sample (from $60^{\circ} \mathrm{C}$ to $25^{\circ} \mathrm{C}$ ).

Figure 4 shows that the transmittance decreases by $36 \%$ with the increase of the temperature. When heating the suspension, the PNIPAAm segments precipitated onto the par- 


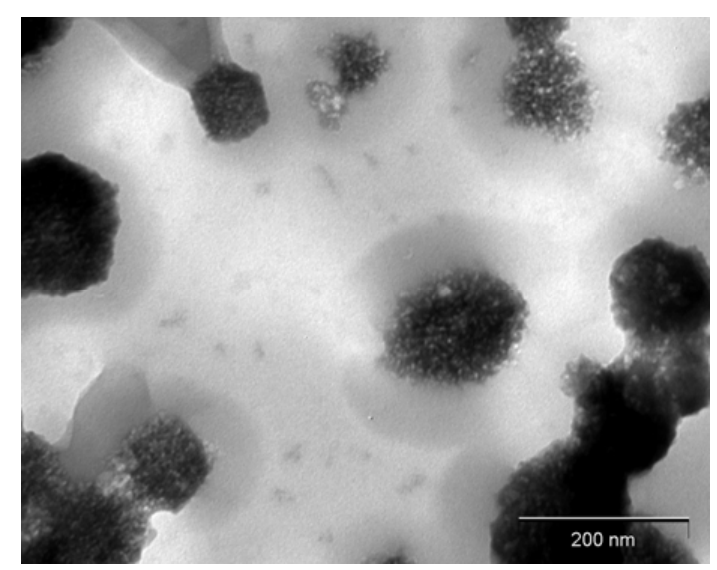

FIG. 3: TEM image of PHBHV-b-PNIPAAm nanoparticles.

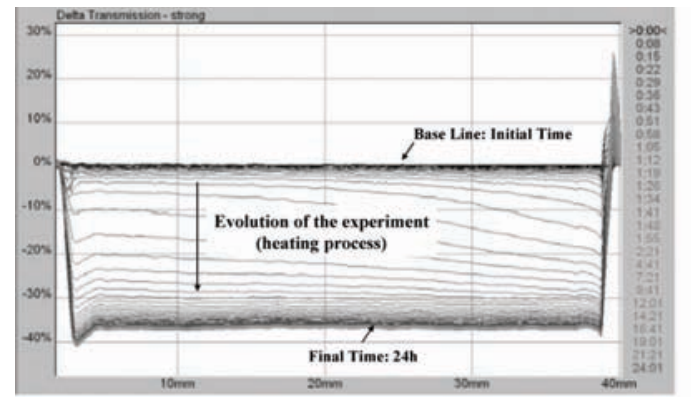

Length of the sample (from the bottom to the top)

FIG. 4: Effect of heating in the transmittance of thermo-responsive nanoparticles suspension $\left(25\right.$ to $\left.60^{\circ} \mathrm{C}\right)$. X-axis represents height of the tube and $\mathrm{Y}$-axis the transmitted light percentage. The values in the column on the right part give the correspondence between the recorded trace and the time elapsed The data are represented as a function of time (0:00 to 24:01 hours) and of sample height (1 to 40 $\mathrm{mm})$.

ticles surface and at these conditions the transmittance was changed.

In contrast, when the sample was cooled from $60^{\circ} \mathrm{C}$ to $25^{\circ} \mathrm{C}$ (Figure 5), the transmittance increased around 36\%. It has occurred due to the increase of solubility of PNIPAAm segments with the decrease of temperature. Important information is that the particle size $\left(D_{p}\right)$ remains unchanged with the heating/cooling cycle, indicating that the nanoparticles coalescence is reversible. By heating the particles suspension, the solubility of PNIPAAm segments in water decreases, allowing the agglomeration of nanoparticles and, when the suspension is cooled again, the hydrophilic segment returns to the original conformation (extended chains). This is a very specific property of nanoparticles obtained by means of selfassembly of amphiphilic block copolymers. This mechanism of agglomeration/deagglomeration allows a very subtle and sophisticated control of the mechanism of drug delivery from nanoparticles. This phenomenon has been usually characterized by means of fluorescence technique [13, 17]. At the best of our knowledge, the utilization of turbidimetry for such purpose has never been reported before in the literature.

A model drug (dexametazone acetate) was encapsulated by

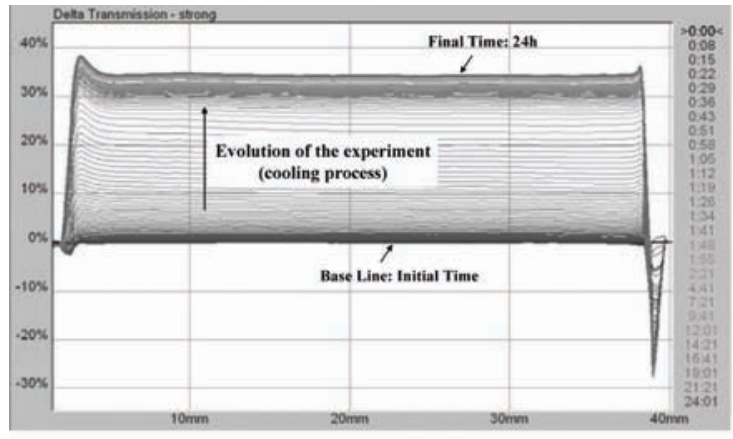

Length of the sample (from the bottom to the top)

FIG. 5: Effect of cooling in the transmittance of thermo-responsive nanoparticles suspension $\left(60\right.$ to $\left.25^{\circ} \mathrm{C}\right)$.

self-assembly technique to study the thermo-responsive property of nanoparticles in drug delivery system. This encapsulation was realized by diffusion of solvent (dialysis) [18]. In this case, the drug was mainly located in the core of nanoparticles due to the physical and chemical affinity between the drug (hydrophobic) and the also hydrophobic PHBHV segments of the block copolymers. The efficiency of the encapsulation was of $77 \%$. The assays of in-vitro drug delivery were realized in phosphate buffer $(p H=7.4)$ at two different temperatures, $4^{\circ} \mathrm{C}$ and $40^{\circ} \mathrm{C}$, respectively, below and above the LCST of the PNIPAAm $\left(32^{\circ} \mathrm{C}\right)$. For comparison, a drug delivery assay was realized at $40^{\circ} \mathrm{C}$ with a sample of free DexAc, i.e., with no encapsulation of the drug by the block copolymer. Figure 6 shows the release kinetics of DexAc from the nanoparticles as a function of the temperature. The DexAc concentration released was determined as previously described in 2.6, using UV/vis spectrometry. As it can be seen, the drug release profile shows drastic changes with temperature alterations, below and above the LCST. In fact, the PNIPAAm segments are located onto the shell of the PHBHV core of the nanoparticles and the thermal sensitivity of the PHBHV-PNIPAAm nanoparticles can control the reversible structural changes of the nanoparticles by temperature cycles through the LCST of PNIPAAm. At $4^{\circ} C$, the highly hydrated PNIPAAm shell stabilizes the drug loaded in the nanoparticle core, allowing a slower diffusion rate. At $40^{\circ} \mathrm{C}$, the PNIPAAm segments collapsed onto the particles surface, facilitating the DexAc diffusion through the outer shell as illustrated in Figure 7.

The oscillating drug release that can be obtained from the PHBHV-PNIPAAm nanoparticles developed in this work is shown in Figure 8, where a fast/slow thermo-responsive switching is controlled by the temperature cycles through the LCST.

\section{CONCLUSION}

Core-shell nanoparticles have been prepared using a biodegradable block of PHBHV (hydrophobic segment) and PNIPAAm, a thermo-responsive polymer (hydrophilic segment), that was synthesized by RAFT polymerization. Selfassembly of PHBHV-b-PNIPAAm in water enabled to encapsulate DexAc, a hydrophobic drug, in order to protect 


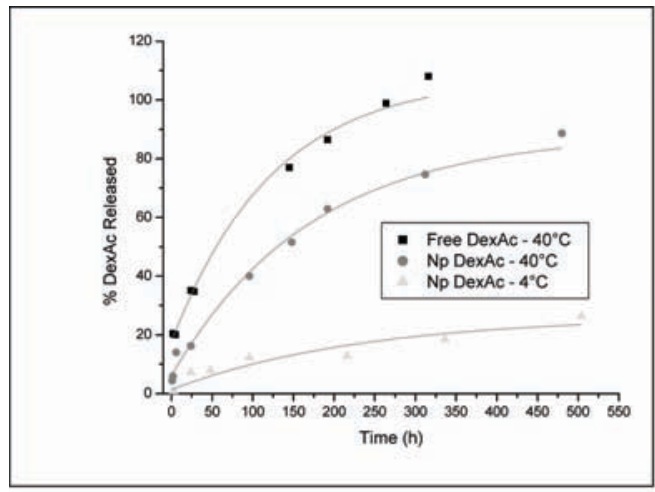

FIG. 6: Drug release from thermo-responsive PHBHV-b-PNIPAAm nanoparticles containing dexametazone acetate. Effect of the temperature above and below the LCST.

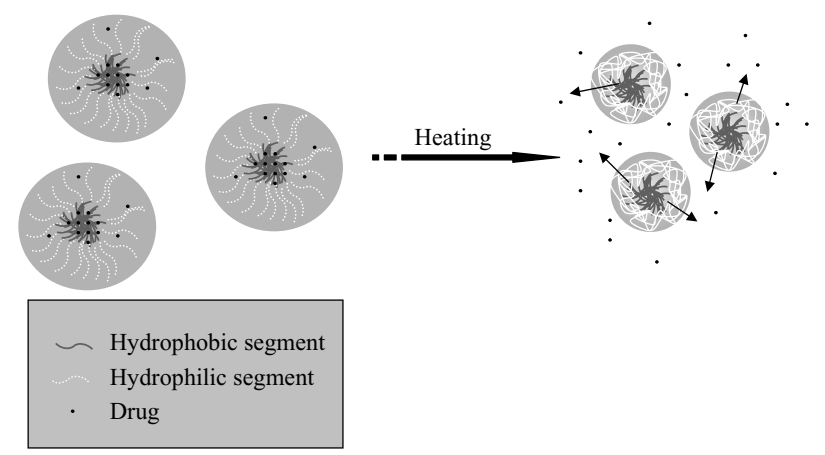

FIG. 7: Schematic illustration of the release mechanism of DexAc from thermo-responsive PHBHV-PNIPAAm nanoparticles. When the temperature is above the LCST of the PNIPAAm, the expanded form of the PNIPAAm in the shell part is changed into the compact one and the drug is ejected. this molecule from the external environment by a hydrophilic PNIPAAm shell. Polymeric micelles loaded with DexAc showed a thermoresponsive behavior. The drug release was strongly dependent on the temperature. This phenomenon was explained by the change in the structure of the micellar shell, with the change of temperature. The particles developed here can be utilized as an "intelligent" drug delivery

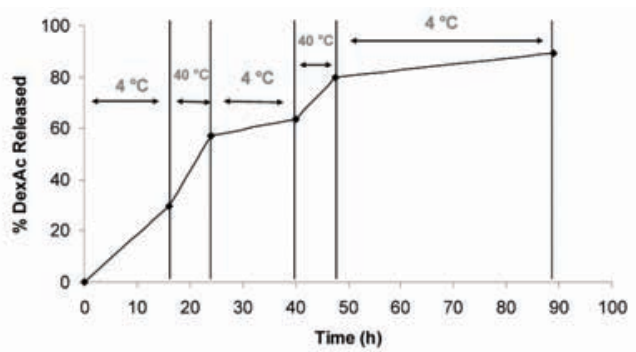

FIG. 8: DexAc released from thermo-responsive and partially biodegradable nanoparticles in response to temperature switching between 4 and $40^{\circ} \mathrm{C}$.

system for site-specific drug release induced by local temperature changes.

\section{Acknowledgements}

The authors are grateful to FAPESP, CNPq and MCT/CNPq for the financial support. The authors also thank P.Y. Dugas, from LCPP/CPE/CNRS (Lyon-France), for the TEM analyses.
[1] N. Tirelli, (Bio)Responsive nanoparticles Current Opinio in Colloid and Interface Science, 11, 210 (2006).

[2] J.Y. KIM; S.B. LEE; S.J. KIM, Y.M. LEE, Rapid temperature/pH reposnse of porous alginate-g-poly $(\mathrm{N}$ isopropylacrylamide) hydrogels Polymer, 43, 7549 (2002).

[3] Y. Hu, Y. Chen, Q. Chen, L. Zhang, X. Jiang, C. Yang, Synthesis and stimuli-responsive properties of chitosan/poly(acrylic acid) hollow nanospheres. Polymer, 46, 12703 (2005).

[4] E.S. Gil, S. M. Hudson, Stimuli-reponsive polymers and their bioconjugates. Prog. Polym. Sci, 29, 1173 (2004).

[5] P. Kujawa, F.M. Winnik, Volumetric studies of aqueous polymer solutions using pressure perturbation calorimetry: a new look at the temperature-induced phase transition of poly $(\mathrm{N}$ isopropylacrylamide) in water and D2O. Macromolecules, 43, 4130 (2001).

[6] M. Heskins, J. E. Guillet, Solution Properties of Poly(Nisopropylacrylamide) J. Macromol. Sci. Chem., A2, 8, 1441 (1968).

[7] M.I.Z. LIONZO, M. I. R, S. S. GUTERRES, A R. POHLMANN, Microparticles prepared with poly(hydroxybutirate-co-hydroxyvalerate) and $\operatorname{poly}(\chi-$ caprolactone) blends to control the release of a drug model, J. of Microencapsulation, 24(2), 175 (2007).

[8] M.I. Ré, M.F.A. Rodrigues, E.S. Silva, I.M. Castro, A. Simioni, M.M.M. Pelisson, J.R.M. Beltrame, A.C. Tedesco, New PHB/PHPE microspheres obtained from Burkholderia cepacia as biodegradable drug delivery systems for photodynamic therapy. Minerva Biotecnologica, 18,(1), 3 (2006)

[9] Y. Yi, and J, Liu, Smart Nanomaterials Inspired by Biology: Dynamic Assembly of Error-Free Nanomaterials in Response to Multiple Chemical and Biological Stimuli. Accounts of Chemical Research, 40(5), 315 (2007).

[10] C. Alen, D. Maysinger, A. Eisenberg, Nano-engineering block copolymer aggregates for drug delivery. Colloids and Surfaces B: Biointerfaces, 16, 3 (1999)

[11] G. Gaucher, M. Dufresne, V. P. Sant, N. Kang, D. Maysinger, J. Leroux, Block copolymer micelles: preparation, characterization and application in drug delivery. J. Controlled Release, 109, 169 (2005).

[12] J. Rodríguez-Hernándes, F. Chécot, Y. Gnanou, S. Lecommandoux, Toward 'smart' nano-objects by self-assembly of block copolymers in solution. Prog. Polym. Sci, 30, 691 (2005).

[13] H. WeI, X. Zhang, Y. Zhou, S. Cheng, R. Zhuo, Self-assembled thermoresponsive micelles of poly(N-isopropylacrylamide-b- 
methyl methacrylate). Biomaterials, 27(I), 2028 (2006).

[14] K. Kataoka, A. Harada, Y. Nagasaki, Block copolymer micelles for drug delivery: desing, characterization and biological significance. Advanced Drug Delivery Reviewers, 47, 113 (2001).

[15] N. Rapoport, Physical stimuli-responsive polymeric micelles for anti-cancer drug delivery, Prog. Polym. Sci. 32, 962 (2007).

[16] L. Zhang, R. Guo, M. Yang, X. Jiang, B. Liu. Thermo and pH Dual-Responsive Nanoparticles for Anti-Cancer. Drug Delivery Advanced Materials, 19, 2988 (2007).

[17] J. E. Chung, M.Yokoyama, T. Aoyagi, Y. Sakurai, T. Okano, Effect of molecular architecture of hydrophobically modified poly( $\mathrm{N}$-isopropylacrylamide) on the formation of thermoresponsive core-shell micellar drug carriers. J. Controll. Rel., 53, 119 (1998)

[18] C. Lo, K. Lin, G. Hsiue, Preparation and characterization of intelligent core-shell nanoparticles based on poly(D,L-lactide)g-poly(N-isopropylacrylamide-co-methacrylic acid). J. Con- trolled Release, 104, 477 (2005)

[19] J. E. Chung, M.Yokoyama, T. Okano, Inner core segment design for drug delivery control of thermo-responsive polymeric micelles. J. Controlled Release, 65, 93 (2000).

[20] F. Kohori, K. Yokoyama, K. Sakai, T. Okano, Process desing for efficient and controlled drug incorporation into polymeric micelle carrier systems. J. Controlled Release, 78, 155 (2002).

[21] F. D’Agosto., R Hughes, M.T. Charreyre, C. Pichot, R.G. Gilbert, Molecular Weight and Functional End Group Control by RAFT Polymerization of a Bisubstituted Acrylamide Derivative. Macromolecules, 36, 621 (2003).

[22] S. Montoro, "Redução da massa molecular do Poli-3Hidroxibutirato-co-3-Hidroxivalerato (PHBHV) para sua posterior utilização no desenvolvimento de sistemas de liberação controlada", MSc Thesis, University of São Paulo, Brazil (2005). 